\title{
Acquirement of Oocyte-activating Factor in Antarctic Minke Whale (Balaenoptera bonaerensis) Spermatogenic Cells, Assessed by Meiosis Resumption of Microinseminated Mouse Oocytes
}

\author{
Kazue Amemiya ${ }^{1}$, Yoshihito Iwanami', Toshihiro Kobayashi ${ }^{2}$, \\ Tatsuma Terao ${ }^{1}$, Yutaka Fukui ${ }^{3}$, Hajime Ishikawa ${ }^{4}$, \\ Seiji Ohsumi ${ }^{4}$, Masumi Hirabayashi ${ }^{5,6}$ and Shinichi Hochi ${ }^{1,2 *}$ \\ ${ }^{1}$ Graduate School of Science and Technology, Shinshu University, 3-15-1 Tokida, Ueda, \\ Nagano 386-8567, Japan \\ ${ }^{2}$ Faculty of Textile Science and Technology, Shinshu University, 3-15-1 Tokida, Ueda, \\ Nagano 386-8567, Japan \\ ${ }^{3}$ Obihiro University of Agriculture and Veterinary Medicine, W2-S11 Inada-cho, Obihiro, \\ Hokkaido 080-8555, Japan \\ ${ }^{4}$ The Institute of Cetacean Research, 4-18 Toyomi-cho, Chuo-ku, Tokyo 104-0055, Japan \\ ${ }^{5}$ National Institute for Physiological Sciences, 5-1 Myodaiji-cho, Okazaki, Aichi 444-8787, Japan \\ ${ }^{6}$ School of Life Science, The Graduate University of Advanced Studies, 5-1 Myodaiji-cho, \\ Okazaki, Aichi 444-8787, Japan
}

\begin{abstract}
The presence or absence of sperm-borne oocyte-activating factor (SOAF) in the Antarctic minke whale haploid spermatogenic cells was determined by assessing the meiosis resumption of microinseminated mouse oocytes. The relative capacity of mature spermatozoa from mouse, cattle and whale to resume the meiosis of BDF1 mouse oocytes was, respectively, $90.5,84.6$ and $76.5 \%$, while nuclear changes in nontreated or buffer-injected oocytes did not occur after 90 min culture. In the whales, the late-stage elongating spermatids as well as the testicular spermatozoa triggered the meiosis resumption of mouse oocytes at similar rates (oocyte activation rates; 68.0 and $62.5 \%$, respectively). The oocyte activating capacity of the early-stage elongating spermatids was significantly lower (25.0\%), and the round spermatids did not activate mouse oocytes at all. This result suggests that the SOAF activity in the Antarctic minke whales is acquired during the early phase of spermiogenesis.
\end{abstract}

Key words: Egg activation, Minke whale, Mouse test, Sperm factor, Spermiogenesis

Received: September 9, 2004

Accepted: September 21, 2004

*To whom correspondence should be addressed.

e-mail: shochi@shinshu-u.ac.jp

\section{Introduction}

The fertilizing of spermatozoa with mammalian oocytes triggers a series of events including release from meiotic arrest, extrusion of the second polar body, DNA replication and the first mitotic cleavage. A transient increase in the intracellular concentration of free calcium $\left(\left[\mathrm{Ca}^{2+}\right]_{i}\right)$ appears to be an up-stream event that activates a cascade of cellular changes necessary for resumption of meiosis and the cell cycle (e.g. inactivation of cell cycle-regulated proteins, such as maturation promoting factor and cytostatic factor). In mammals but not Xenopus, fertilization starts the repetitive $\left[\mathrm{Ca}^{2+}\right]_{i}$ transients, the so-called $\left[\mathrm{Ca}^{2+}\right]_{i}$ oscillations [1], but the signaling mechanism by which the sperm starts the $\left[\mathrm{Ca}^{2+}\right]_{i}$ oscillations has not been fully understood. One possible hypothesis to take into account the mechanism is that a soluble substance from the fertilizing sperm, named sperm-borne oocyteactivating factor (SOAF), is delivered into the ooplasm and triggers the oocyte activation [2]. A promising candidate for the SOAF may be one of the phospholipase $C$ isoforms that plays a crucial role in phosphoinositide turnover by hydrolyzing 
phosphatidylinositol-4,5-bisphosphate to generate two second messengers, inositol 1,4,5-triphosphate and diacylglycerol [3, 4].

Homologous IVF or microinsemination is the most convenient approach to detect the SOAF activity in spermatogenic cells [2, 5], but may be difficult to achieve in some species (e.g. dog, horse and human). Because of very little specificity in the SOAF activity between species [6-11], mammalian spermatozoa can be experimentally microinjected into mouse oocytes to assess their oocyte-activating capacity. The accumulated results of the homologous and interspecies assay indicate that the stage at which the SOAF first emerges or becomes biologically active during spermatogenesis may vary with species. For example, in primates such as human [5] and cynomolgus monkey [11], the SOAF is already present at the round spermatid stage, as demonstrated by their oocyte-activating capacity and the generation of $\left[\mathrm{Ca}^{2+}\right]_{i}$ oscillations after intracytoplasmic injection. It was also reported that the SOAF activity is present in hamster and rabbit round spermatids [10]. Normal human babies [12] and hamster offspring [13] have been produced after round spermatid injection without the aid of artificial stimuli to activate the oocytes. By contrast, there is no oocyte-activating capacity in the round spermatids of mouse $[9,10]$ and rat [10]. The SOAF activity of mouse elongating spermatids was intermediate between that of round spermatids and that of mature spermatozoa [9].

In marine mammals (e.g. dolphins and whales), literature associated with the gamete interaction during fertilization is very limited [14-17], probably due to the insufficient availability of the experimental materials. Only the early cleavages of minke whale oocytes after IVF $[14,16]$ and intracytoplasmic sperm injection (ICSI) [17] have been reported. The aim of the present study is to expand fundamental knowledge of reproductive physiology, especially SOAF acquirement during spermatogenesis, in minke whales (Balaenoptera bonaerensis). Since the Japanese Whale Research Program under Special Permit in the Antarctic Sea (International Whale Commission-approved) allowed us to use testicular samples from the minke whales captured in the non-breeding season (December 2003 to January 2004), we report here the timing at which whale spermatogenic cells acquire the SOAF activity, by assessing the meiosis resumption of microinseminated mouse oocytes.

\section{Materials and Methods}

\section{Experimental design}

In Experiment 1, mature spermatozoa from mouse cauda epididymis, bull ejaculate and minke whale deferent duct were microinjected into $F_{1}$ hybrid mouse oocytes after dissociation of the sperm tail. The meiosis resumption of the ICSI oocytes was evaluated 90 min after the injection. Both oocytes injected with the buffer alone and oocytes left for $90 \mathrm{~min}$ in culture served as the negative controls (buffer-injected group and nontreated group, respectively). In Experiment 2, spermatogenic cells from adult minke whales were microinjected into mouse oocytes, and the meiosis resumption of the microinseminated oocytes was also evaluated. The spermatogenic cells include round spermatids (Gorgi/cap phase, stages 1-7), early-stage elongating spermatids (acrosome phase, stages 8-14), late-stage elongating spermatids (maturation phase, stages 15-19) and testicular spermatozoa [18].

\section{Preparation of sperm/spermatids}

Mature spermatozoa for Experiment 1: (1) Spermatozoa from cauda epididymis of a Crj:BDF1 male mouse (Charles River Japan, Kanagawa, Japan) were suspended at $4^{\circ} \mathrm{C}$ in the Dulbecco's phosphatebuffered saline (PBS) containing $5.6 \mathrm{mM}$ glucose, 5.4 $\mathrm{mM}$ sodium lactate and $0.1 \%$ polyvinyl pyrrolidone (360 KDa; Sigma-Aldrich Chemicals, St. Louis, MO, USA), referred to hereinafter GL-PBS. The sperm suspension was centrifuged twice at $200 \mathrm{~g}$ for $5 \mathrm{~min}$ at $4{ }^{\circ} \mathrm{C}$ and cryopreserved in the GL-PBS supplemented with $7.5 \%$ glycerol (Wako Pure Chemical Industries, Osaka, Japan) and $7.5 \%$ fetal bovine serum (FBS; Equitech Bio, Ingram, TX, USA) in 1.0-ml cryotubes, according to the method by Ogura et al. [19]. The temperature of the water bath to warm the frozen tubes was $20^{\circ} \mathrm{C}$. The post-warm samples were washed twice by centrifugation at $200 \mathrm{~g}$ for $5 \mathrm{~min}$ with the GL-PBS. (2) Spermatozoa from a Japanese Black bull were derived from the commercially available semen frozen in an egg yolk-Tris buffer/glycerol solution in $0.5-\mathrm{ml}$ straws. The samples thawed in a $35^{\circ} \mathrm{C}$ water bath were washed twice by centrifugation at $490 \mathrm{~g}$ for 5 min with Brackett and Oliphant solution [20]. (3) Spermatozoa from a minke whale were recovered by squeezing the deferent ducts downstream, and suspended in an egg yolk-Tris buffer/glycerol solution in 1.0-ml Eppendorf tubes. The tubes were cooled to $5^{\circ} \mathrm{C}$ for $2 \mathrm{~h}$ and to $-80^{\circ} \mathrm{C}$ overnight, and then stored in liquid nitrogen, according to the method by Fukui et al. [21]. The temperature of 
the water bath to warm the frozen tubes was $37^{\circ} \mathrm{C}$, and the post-warm samples were washed twice by centrifugation at $200 \mathrm{~g}$ for $5 \mathrm{~min}$ with the GL-PBS.

Whale spermatogenic cells for Experiment 2: Testicular spermatogenic cells were prepared from pubertal minke whales on the research base ship. Briefly, cubic centimeter testis clods were placed in the erythrocyte-lysing buffer $\left(155 \mathrm{mM} \mathrm{NH}_{4} \mathrm{Cl}, 10 \mathrm{mM}\right.$ $\mathrm{KHCO}_{3}, 2 \mathrm{mM}$ EDTA; $\mathrm{pH} 7.2$ ). Seminiferous tubules were transferred into the GL-PBS at ambient temperature, cut into small pieces with fine scissors, and shaken gently to release spermatogenic cells into the medium. The cell suspension was filtered through a 40- $\mu \mathrm{m}$ nylon mesh and then centrifuged at $200 \mathrm{~g}$ for 5 min. The suspension containing the spermatids and spermatozoa in cryotubes was cryopreserved in the GLPBS supplemented with $7.5 \%$ glycerol and $7.5 \%$ FBS, as described above for the mature mouse spermatozoa. The tubes were warmed in a $20^{\circ} \mathrm{C}$ water bath immediately before injection, and resuspended with 10 fold volumes of the GL-PBS.

\section{Morphology of sperm/spermatids}

Mature spermatozoa from mouse, cattle and whale were double-stained [22]. Briefly, spermatozoa after being air-dried on coverslips were stained with $0.1 \%$ Naphthol-yellow-S (Wako) in diluted acetic acid ( $\mathrm{pH} 2.8$ ) for $30 \mathrm{~min}$, soaked in $0.1 \%$ acetic acid for $8 \mathrm{sec}$, and washed with ultra pure water. They were then stained with $0.2 \%$ Naphthol-yellow-S and $0.2 \%$ Erythrosin-B (Wako) in diluted acetic acid ( $\mathrm{pH} \mathrm{4.8)}$ for $15 \mathrm{~min}$, and rinsed with the solvent.

Spermatogenic cells from whales were observed under a laser-scanning confocal microscope (BIO-RAD Radiance 2000/KR2; Bio-Rad, Tokyo, Japan) [23] with a few minor modifications. Briefly, cells were allowed to attach to the coverslips coated with $0.1 \%$ poly-L-lysine (Sigma-Aldrich), fixed with $4 \%$ formaldehyde for $10 \mathrm{~min}$, and washed with the PBS. After being treated with $1 \%$ Triton X-100 for $5 \mathrm{~min}$ and washed with the PBS, the cells were incubated for $10 \mathrm{~min}$ in the blocking buffer (PBS containing 2\% BSA and $130 \mathrm{mM}$ glycine). The cells were incubated for $60 \mathrm{~min}$ with the antibody against $\alpha$-tubulin (T5168; Sigma-Aldrich), blocked for 10 min, and then treated with the FITC-tagged second antibody against mouse IgG (F1010; Sigma-Aldrich) for $60 \mathrm{~min}$. The cells were stained with $5 \mu \mathrm{g} / \mathrm{ml}$ propidium iodide (PI; Sigma-Aldrich) for $5 \mathrm{~min}$, and then rinsed with the PBS.

\section{Mouse oocyte activation assay}

Oocyte preparation: The specific pathogen-free/virus antibody-free Crj:BDF1 female mice (7-10 weeks old; Charles River Japan) were superovulated by intraperitoneal injections of $5 \mathrm{i}$.u. equine chorionic gonadotropin (eCG; Denka Pharmaceuticals, Kanagawa, Japan) and 5 i.u. human chorionic gonadotropin (hCG; Mochida Pharmaceuticals, Tokyo, Japan) at an interval of $48 \mathrm{~h}$. The oocytes were collected from the oviductal ampullae $14 \mathrm{~h}$ after the hCG injection and were freed from cumulus cells by a 5 min treatment with $0.1 \%$ hyaluronidase (Sigma-Aldrich) suspended in the CZB medium [24]. The denuded oocytes were rinsed and incubated in CZB medium for up to $2 \mathrm{~h}$ at $37^{\circ} \mathrm{C}$ in an atmosphere of $5 \% \mathrm{CO}_{2}$ in air.

Sperm injection: Prior to the ICSI, 1.7- $\mu$ l of the sperm suspension was mixed with $8.3-\mu$ l of the CZB medium supplemented with $22 \mathrm{mM}$ Hepes and 12\% PVP, defined hereafter to as Hepes-CZB/PVP medium, at an ambient temperature of $23 \pm 2^{\circ} \mathrm{C}$. Using a piezo-impact driving unit (PMM-140FU; Prime Tech, Ibaraki, Japan) with a pulse controller (PMAS-CT140; Prime Tech), the sperm head was dissociated from the tail by a few piezo pulses. Ten denuded oocytes were placed in $10-\mu l$ of the CZB medium supplemented with $22 \mathrm{mM}$ Hepes, referred to hereinafter Hepes-CZB medium. The sperm head was then deposited in an ooplasm with a bluntended injection pipette with an inner diameter of 5-6 $\mu \mathrm{m}$.

Spermatid injection: The round and elongating spermatids were suspended in $10-\mu$ of the Hepes-CZB/ PVP medium. The procedure for spermatid injection was according to the method described by Hirabayashi et al. [25]. Briefly, the spermatid's membrane was broken by shear stress during the repeated pipetting. The spermatid nucleus with the surrounding cytoplasm was then microinjected into an ooplasm in the HepesCZB medium (10 oocytes per $10-\mu$ l microdrop).

\section{Assessment of SOAF presence}

The microinseminated oocytes as well as the control oocytes were incubated in $100-\mu l$ microdrops of the CZB medium for at least $90 \mathrm{~min}$ at $37^{\circ} \mathrm{C}$ in an atmosphere of $5 \% \mathrm{CO}_{2}$ in air. The oocytes were placed on a slide glass, covered with a coverslip, and then fixed with acetic acid:ethanol (1:3) for $24 \mathrm{~h}$. The oocytes stained with $1 \%$ aceto-orcein for 5 min were observed through a phase-contrasted microscope at $\times 200$ magnification. The oocytes with the anaphase-II or telophase-II plate were defined as resumed meiosis (activated). 


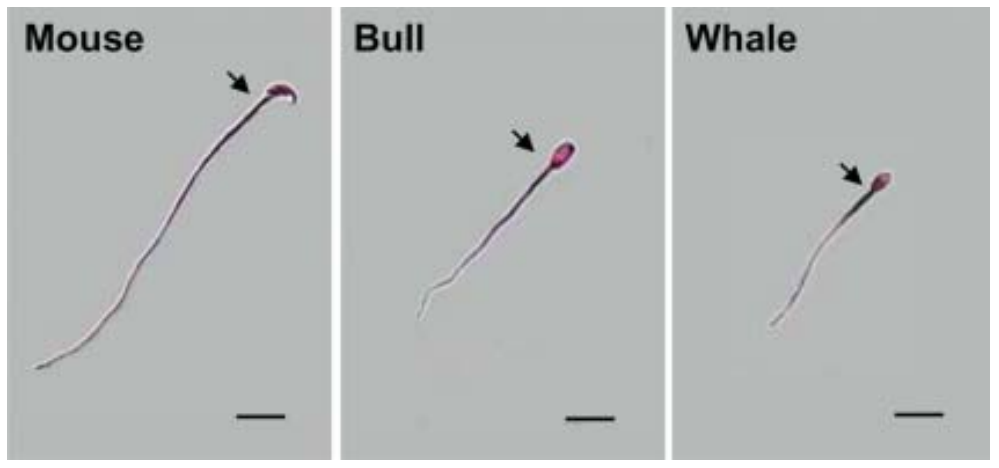

Fig. 1. Morphology of mature spermatozoa derived from mouse, bull and minke whale after double-staining (bar $=10 \mu \mathrm{m}$ ). Each arrow indicates the site dissociated by piezo pulses prior to ICSI.

Table 1. Activation of mouse oocytes by microinjected mouse, bull and minke whale spermatozoa

\begin{tabular}{lcc}
\hline \multicolumn{1}{c}{ Groups } & No. of oocytes injected & No. of oocytes activated \\
\hline Mouse spermatozoa & 42 & $38(90.5 \%)^{\mathrm{a}}$ \\
Bull spermatozoa & 39 & $33(84.6 \%)^{\mathrm{a}}$ \\
Whale spermatozoa & 51 & $39(76.5 \%)^{\mathrm{a}}$ \\
Buffer-injected control & 50 & $0(0 \%)^{\mathrm{b}}$ \\
Non-treated control & 38 & $0(0 \%)^{\mathrm{b}}$
\end{tabular}

${ }^{\mathrm{a}, \mathrm{b}}$ Different superscripts denote significant difference at $\mathrm{P}<0.05$.

\section{Statistical analysis}

The proportions of activated oocytes among experimental groups were compared by the Lyanmethod [26]. A value of $P<0.05$ was chosen as an indication of statistical significance.

\section{Results}

\section{Experiment 1}

The morphology of the mature spermatozoa from the three species (mouse, cattle and whale) is shown in Fig. 1. There were species-specific characteristics in the shape of the sperm head and mid-piece. The whale spermatozoon (c.a. $60 \mu \mathrm{m}$ whole length) was much shorter than the mouse spermatozoon $(130 \mu \mathrm{m})$ and slightly shorter than the bull spermatozoon $(70 \mu \mathrm{m})$. The sites at which piezo pulses were given to dissociate sperm heads from the tails are shown on the same photographs.

The result of mouse oocyte activation assay with these sperm heads is shown in Table 1. In the two negative control groups, none of the BDF1 mouse oocytes resumed meiosis either spontaneously in 90 min culture or mechanically by injecting with the buffer alone. The proportions of mouse oocytes activated by the injection of mouse, bull and whale sperm heads were $90.5,84.6$ and $76.5 \%$, respectively $(P>0.05)$.

\section{Experiment 2}

The typical confocal microphotographs of whale spematogenic cells representing both cytoskeletal and nuclear appearances are shown in Fig. 2. Diameters of the spherical round spermatid (RS) and their nucleus were $12-15 \mu \mathrm{m}$ and $7-8 \mu \mathrm{m}$, respectively. The round spermatid transformed into the early-stage elongating spermatid (e-ES), the late-stage elongating spermatid (I-ES), and then the spermatozoon (SP) during the spermiogenesis. The $\alpha$-tubulin distributed homogenously in the cytoplasm of the round and elongating spermatids, and localized in the mid-piece and tail of the spermatozoon.

The result of mouse oocyte activation assay with the spermatogenic cells is shown in Table 2. The late-stage elongating spermatids and the testicular spermatozoa triggered the meiosis resumption of mouse oocytes at similar rates to the oocyte activation rates which were 68.0 and $62.5 \%$, respectively $(P>0.05)$. The proportion of oocytes activated by the early-stage elongating 

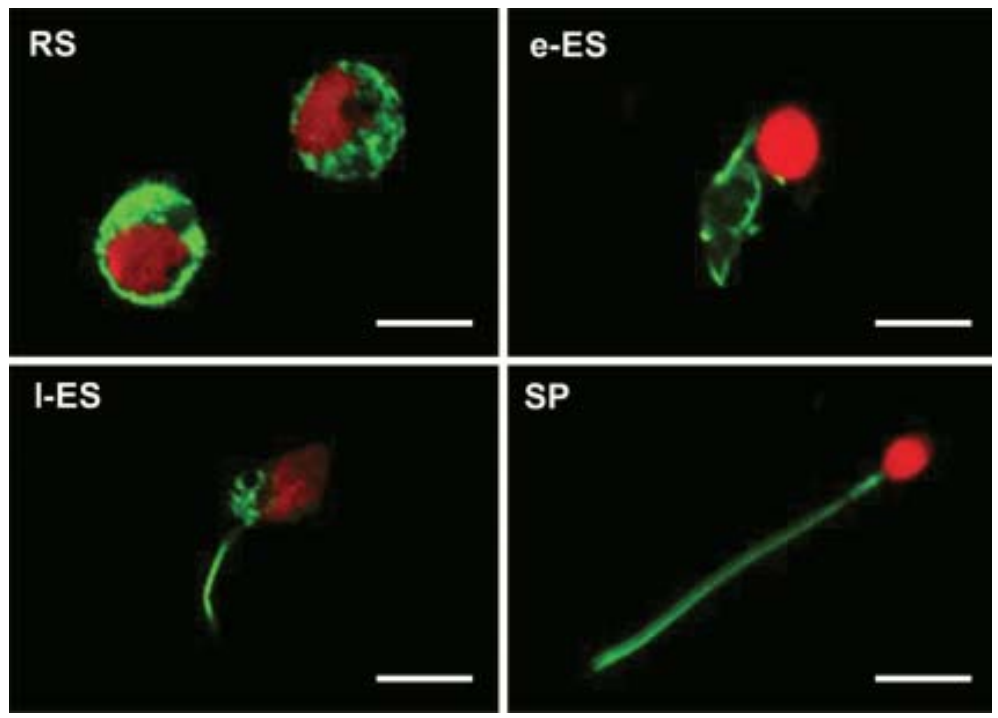

Fig. 2. Minke whale testis-derived spermiogenic cells after PI-staining and immuno-staining against $\alpha$-tubulin (bar $=10 \mu \mathrm{m}$ ). Two fluorescent images of cell nucleus under $514 \mathrm{~nm}$ UV light (red) and $\alpha$-tubulin under $488 \mathrm{~nm}$ UV light (green) were combined with a program of LaserPix (Bio-Rad). RS: round spermatids (Gorgi/cap phase), e-ES: early-stage elongating spermatids (acrosome phase), l-ES: late-stage elongating spermatids (maturation phase), SP: spermatozoa.

Table 2. Stage-dependent acquirement of oocyte-activating factor during spermiogenesis in the minke whale

\begin{tabular}{lcc}
\hline \multicolumn{1}{c}{ Group* } & No. of oocytes injected & No. of oocytes activated \\
\hline Round spermatids & 48 & $0(0 \%)^{\mathrm{c}}$ \\
Early-stage elongating spermatids & 52 & $13(25.0 \%)^{\mathrm{b}}$ \\
Late-stage elongating spermatids & 50 & $34(68.0 \%)^{\mathrm{a}}$ \\
Testicular spermatozoa & 48 & $30(62.5 \%)^{\mathrm{a}}$ \\
\hline
\end{tabular}

*Refer to Fig. 2. ${ }^{\mathrm{a}-\mathrm{c}}$ Different superscripts denote significant difference at $\mathrm{P}<0.05$.

spermatids $(25.0 \%)$ was significantly lower than those activated by the late-stage elongating spermatids and testicular spermatozoa $(P<0.05)$. The round spermatids did not activate mouse oocytes at all $(0 \%, \mathrm{P}<0.05$; versus the other 3 groups).

\section{Discussion}

The poorly established regimens for IVF or microinsemination in whales [14-17] and the limited availability of whale oocytes led us to use the interspecies microinsemination assay for SOAF detection in the spermatogenic cells. This alternative experimental system with mouse oocytes has been demonstrated to be effective for the spermatozoa of several mammalian species including small rodents, large domestic animals and primates $[6-8,10,11]$. The strain of the donor mouse is important for successful mouse oocyte activation assay because meiosis resumption of oocytes from some strains occurs spontaneously in vitro [27] or after a mechanical shamoperation [28]. Ovulated oocytes from hamster [29] and in vitro-matured oocytes from pig [30] and cattle [31] are used for the same purpose. The assay would have more advantages in SOAF research on not only some animal species in which the conventional IVF and microinsemination techniques are still under investigation (e.g. dog and horse) but also endangered wild species (including marine mammals) and human in which sufficient numbers of homologous oocytes are 
theoretically or ethically difficult to collect. Wei and Fukui [15] reported that approximately $30 \%$ of bovine oocytes microinjected with minke whale spermatozoa had male and female pronuclei $18 \mathrm{~h}$ after the ICSI.

There seem to be two groups with different timing in which the SOAF first emerges or becomes biologically active during spermatogenesis by the species. The SOAF in mouse $[9,10,32,33]$ and rat $[9,10]$ is not detectable at the round spermatid stage but it is gradually acquired as the spermatids are elongated. By contrast, in hamster [10,33], rabbit [10], cynomolgus monkey [11] and human [5, 7, 10, 33], the SOAF is already present at the round spermatid stage (or possibly the earlier spermatocyte stage in primates). The birth of human babies [12] and hamster offspring [13] from round spermatid-injected oocytes without the aid of artificial activation stimuli supports the result of interspecies microinsemination assay [5, 7, 10, 32], as far as the mechanical stimuli applied to the oolemma/ ooplasm do not activate the homologous oocytes. In the present study (Table 2), the SOAF activity of earlystage elongating whale spermatids $(25.0 \%$ as the proportion of mouse oocytes activated) was intermediate between that of round spermatids $(0 \%)$ and that of late-stage elongating spermatids or testicular spermatozoa (62.5-68.0\%), suggesting that the minke whale is one of the constituents of the former group. In addition, the morphological characteristics of the whale spermiogenic cells under confocal microscopy were described for the first time (Fig. 2). Being compared with round spermatids in mouse [18], rat [22] and cynomolgus monkey [11], the whale round spermatids appear to have diameters comparable to the whole cell and nucleus, while the length of the mouse sperm tail was much longer than that of the whale sperm tail (Fig. 1).

The SOAF activity of whale mature spermatozoa (76.5\%; Table 1) cannot be compared with those of mouse and bull spermatozoa (90.5 and $84.6 \%$, respectively), since the loci of the male genital tract where the mature spermatozoa were recovered differed according to the species and only one individual was used as a donor for the spermatozoa in Experiment 1. Due to the different individuals, whether the SOAF activity of whale deferent ductal mature spermatozoa (76.5\%; Table 1) is really higher than that of testicular spermatozoa (62.5\%; Table 2) was not clarified. These problems and the related possibilities remain to be explained. In addition, the question of whether the SOAF activity of spermatogenic cells in mouse oocytes may not be comparable with that in homologous oocytes has been raised. In human [33] and rabbit [a personal communication with Dr. Ogonuki, N. of RIKEN, Tsukuba, Japan], the SOAF activity of spermatogenic cells was different between the mouse oocyte activation assay and the homologous microinsemination assay. The active potential of SOAF in mouse oocytes may be not so high. Since the whale oocytes appear to be dark due to the much cytoplasmic lipid droplets [14, 16, 17], dispersion of the SOAF molecules throughout the ooplasm is more or less disturbed. Assisting oocyte activation in the whale ICSI may be effective for embryo production as reported in the ICSI of bovine [34, 35] and equine species [36]. Nevertheless, Asada et al. [17] reported that only 2 out of 44 frozen-thawed and ethanol-treated whale oocytes into which the dithiothreitol-treated whale spermatozoa were injected developed to the 2-cell stage.

The generation of $\left[\mathrm{Ca}^{2+}\right]_{i}$ oscillations after intracytoplasmic injection of whale spermatogenic cells was not examined in the present study. Ogonuki et al. [11] reported that cynomolgus monkey spermatocytes had the activating capacity of mouse oocytes without the typical kinetics of $\left[\mathrm{Ca}^{2+}\right]_{i}$ oscillations. Yazawa et al. [10] reported that only a few $\left[\mathrm{Ca}^{2+}\right]_{i}$ transients were observed in mouse oocytes into which the earliest immature sperm cells carrying SOAF activity were microinjected. Although a transient increase in $\left[\mathrm{Ca}^{2+}\right]_{i}$ is an obvious trigger of the oocyte activation [37-39], the $\left[\mathrm{Ca}^{2+}\right]_{i}$ kinetics induced by whale SOAF and its physiological significance in activating homologous oocytes remained to be clarified. The action of the SOAF will be determined more precisely if the molecule is identified. From the recent publications [3, 40], phospholipase $\mathrm{C} \zeta$ may be a promising candidate for mammalian SOAF.

In conclusion, the mouse oocyte activation assay employed here indicates that the spermatogenic cells of the Antarctic minke whale acquire the oocyte-activating capacity at the relatively early elongating spermatid stage. The initial timing of SOAF acquirement in the whale was similar to that in mouse and rat, but much later than that in hamster, rabbit, monkey, and human.

\section{Acknowledgements}

The authors thank the Captain and crew of the research base ship Nisshin-maru for their help in obtaining the Antarctic minke whale testes. 


\section{References}

1) Stricker, S.A. (1999): Comparative biology of calcium signaling during fertilization and egg activation in animals. Dev. Biol., 211, 157-176.

2) Swann, K. (1990): A cytosolic sperm factor stimulates repetitive calcium increases and mimics fertilization in hamster eggs. Development, 110, 1295-1302.

3) Saunders, C.M., Larman, M.G., Parrington, J., Cox, L.J., Royse, J., Blayney, L.M., Swann, K. and Lai, F.A. (2002): PLC $\zeta$ : a sperm-specific trigger of $\mathrm{Ca}^{2+}$ oscillations in eggs and embryo development. Development, 129, 3533-3544.

4) Kurokawa, M., Sato, K. and Fissore, R.A. (2004): Mammalian fertilization: from sperm factor to phospholipase C $\zeta$. Biol. Cell, 96, 37-45.

5) Sousa, M., Mendoza, C., Barros, A. and Tesarik, J. (1996): Calcium responses of human oocytes after intracytoplasmic injection of leukocytes, spermatocytes and round spermatids. Mol. Hum. Reprod., 2, 853-857.

6) Stice, S.L. and Robl, J.M. (1990): Activation of mammalian oocytes by a factor obtained from rabbit sperm. Mol. Reprod. Dev., 25, 272-280.

7) Rybouchkin, A., Dozortsev, D., De Sutter, P., Qian, C. and Dhont, M. (1995): Intracytoplasmic injection of human spermatozoa into mouse oocytes: a useful model to investigate the oocyte-activating capacity and the karyotype of human spermatozoa. Hum. Reprod., 10, $1130-1135$.

8) Wu, H., He, C.L. and Fissore, R.A. (1997): Injection of a porcine sperm factor triggers calcium oscillations in mouse oocytes and bovine eggs. Mol. Reprod. Dev., 46, 176-189.

9) Kimura, Y., Yanagimachi, R., Kuretake, S., Bortkiewicz, H., Perry, A.C.F. and Yanagimachi, H. (1998): Analysis of mouse oocyte activation suggests the involvement of sperm perinuclear material. Biol. Reprod., 58, 1407-1415.

10) Yazawa, H., Yanagida, K., Katayose, H., Hayashi S. and Sato, A. (2000): Comparison of oocyte activation and $\mathrm{Ca}^{2+}$ oscillation-inducing abilities of round/elongated spermatids of mouse, hamster, rat, rabbit and human assessed by mouse oocyte activation assay. Hum. Reprod., 15, 25822590 .

11) Ogonuki, N., Sankai, T., Yagami, K., Shikano, T., Oda, S., Miyazaki, S. and Ogura, A. (2001): Activity of a spermborne oocyte-activating factor in spermatozoa and spermatogenic cells from cynomolgus monkeys and its localization after oocyte activation. Biol. Reprod., 65, 351357.

12) Tesarik, J., Mendoza, C. and Testart, J. (1995): Viable embryos from injection of round spermatids into oocytes. New Engl. J. Med., 333, 525.

13) Haigo, K., Yamauchi, Y., Yazama, F., Yanagimachi, R. and Horiuchi, T. (2004): Full-term development of hamster embryos produced by round spermatids injection into oocytes. Biol. Reprod., 71, 194-198.

14) Fukui, Y., Mogoe, T., Ishikawa, H. and Ohsumi, S. (1997): In vitro fertilization of in vitro matured minke whale (Balaenoptera acutorostrata) follicular oocytes. Mar.
Mamm. Sci., 13, 395-404.

15) Wei, H. and Fukui, Y. (2000): Fertilisability of ovine, bovine or minke whale (Balaenoptera acutorostrata) spermatozoa intracytoplasmically injected into bovine oocytes. Zygote, 8, 267-274.

16) Asada, M., Tetsuka, M., Ishikawa, H., Ohsumi, S. and Fukui, Y. (2001): Improvement on in vitro maturation, fertilization and development of minke whale (Balaenoptera bonaerensis) oocytes. Theriogenology, 56, 521-533.

17) Asada, M., Wei, H., Nagayama, R., Tetsuka, M., Ishikawa, H., Ohsumi, S. and Fukui, Y. (2001): An attempt at intracytoplasmic sperm injection of frozen-thawed minke whale (Balaenoptera bonaerensis) oocytes. Zygote, 9, 299-307.

18) Leblond, C.P. and Clermont, Y. (1952): Definition of the stages of the cycle of the seminiferous epithelium in the rat. Ann. N.Y. Acad. Sci., 55, 548-573.

19) Ogura, A., Matsuda, J., Asano, T., Suzuki, O. and Yanagimachi, R. (1996): Mouse oocytes injected with cryopreserved round spermatids can develop into normal offspring. J. Assist. Reprod. Genet., 13, 431-434.

20) Brackett, B.G. and Oliphant, G. (1975): Capacitation of rabbit spermatozoa in vitro. Biol. Reprod., 12, 260-274.

21) Fukui, Y., Mogoe, T., Ishikawa, H. and Ohsumi, S. (1998): Effect of diluent composition and temperature on motility and viability after liquid storage and cryopreservation of minke whale (Balaenoptera acutorostrata) spermatozoon. Mar. Mamm. Sci., 14, 854-860.

22) Lenz, R.W., Ball. G.D., Lohse, J.K., First, N.L. and Ax, R.L. (1983): Chondroitin sulfate facilitates an acrosome reaction in bovine spermatozoa as evidenced by light microscopy, electron microscopy and in vitro fertilization. Biol. Reprod., 28, 683-690.

23) Moreno, R.D. and Shatten, G. (2000): Microtubule configurations and post-translational $\alpha$-tubulin modifications during mammalian spermatogenesis. Cell Motil. Cytoskeleton, 46, 235-246.

24) Chatot, C.L., Lewis, J.L., Torres, I. and Ziomek, A. (1990): Development of 1-cell embryos from different strains of mice in CZB medium. Biol. Reprod., 42, 432-440.

25) Hirabayashi, M., Kato, M., Aoto, T., Ueda, M. and Hochi, S. (2002): Rescue of infertile transgenic rat lines by intracytoplasmic injection of cryopreserved round spermatids. Mol. Reprod. Dev., 62, 295-299.

26) Ryan, T.A. (1960): Significance tests for multiple comparisons of proportions, variances, and other statistics. Psychol. Bull., 57, 318-328.

27) Eppig, J.J., Wigglesworth, K. and Hirao, Y. (2000): Metaphase I arrest and spontaneous parthenogenetic activation of strain LTXBO oocytes: chimeric reaggregated ovaries establish primary lesion in oocytes. Dev. Biol., 224, 60-68.

28) Yazawa, H., Yanagida, K. and Sato, A. (2001): Oocyte activation and $\mathrm{Ca}^{2+}$ oscillation-inducing abilities of mouse round/elongated spermatids and the developmental capacities of embryos from spermatid injection. Hum. 
Reprod., 16, 1221-1228.

29) Goud, P.T., Goud, A.P., Rybouchkin, A.V., De Sutter, P. and Dhont, M. (1998): Chromatin decondensation, pronucleus formation, metaphase entry and chromosome complements of human spermatozoa after intracytoplasmic sperm injection into hamster oocytes. Hum. Reprod., 13, $1336-1345$.

30) Kim, N.H., Jun, S.H., Do, J.T., Uhm, S.J. and Lee, H.T. (1999): Intracytoplasmic injection of porcine, bovine, mouse or human spermatozoon into porcine oocytes. Mol. Reprod. Dev., 53, 84-91.

31) Smith, J.F. and Murray, G.R. (1996): Use of bovine oocytes for the evaluation of ram semen. Proc. N.Z. Soc. Anim. Prod., 25, 304-306.

32) Sakurai, A., Oda, S., Kuwabara, Y. and Miyazaki, S. (1999): Fertilization, embryonic development, and offspring from mouse eggs injected with round spermatids combined with $\mathrm{Ca}^{2+}$ oscillation-inducing sperm factor. Mol. Hum. Reprod., 5, 132-138.

33) Yanagida, K., Yazawa, H., Katayose, H., Kimura, Y., Hayashi, S. and Sato, A. (2000): Oocyte activation induced by spermatids and the spermatozoa. Int. J. Androl., 23 (Suppl 2), 63-65.

34) Rho, G.J., Wu, B., Kawarsky, S., Leibo, S.P. and Betteridge, K.J. (1998): Activation regimens to prepare bovine oocytes for intracytoplasmic sperm injection. Mol. Reprod. Dev., 50, 485-492.

35) Chung, J.T., Keefer, C.L. and Downey, B.R. (2000) Activation of bovine oocytes following intracytoplasmic sperm injection (ICSI). Theriogenology, 53, 1273-1284.

36) Li, X., Morris, L.H. and Allen, W.R. (2000): Effects of different activation treatments on fertilization of horse oocytes by intracytoplasmic sperm injection. J. Reprod. Fertil., 119, 253-260.

37) Nagai, T. (1987): Parthenogenetic activation of cattle follicular oocytes in vitro with ethanol. Gamete Res., 16, 243-249.

38) Collas, P., Sullivan, E.J. and Barnes, F.L. (1993): Histone H1 kinase activity in bovine oocytes following calcium stimulation. Mol. Reprod. Dev., 34, 224-231.

39) Tesarik, J. and Sousa, M. (1995): More than $90 \%$ fertilization rates after intracytoplasmic sperm injection and artificial induction of oocyte activation with calcium ionophore. Fertil. Steril., 63, 343-349.

40) Cox, L.J., Larman, M.G., Saunders, C.M., Hashimoto, K., Swann, K. and Lai, F.A. (2002): Sperm phospholipase C $\zeta$ from humans and cynomolgus monkeys triggers calcium oscillations, activation and development of mouse oocytes. Reproduction, 124, 611-623. 